section elected Professor Winslow Upton, Ladd Observatory, as member of the sectional committee for five years.

G. A. Miller, Secretary.

\title{
SOME SURFACES HAVING A FAMILY OF HELICES AS ONE SET OF LINES OF CURVATURE.*
}

BY MISS EVA M. SMITH.

IN a recent paper, Forsyth $\dagger$ gives a general method for the determination of surfaces with assigned lines of curvature, and he solves completely the case where both sets are circles. We apply the method to the case where one of the given sets consists of helices, and it appears that surfaces do exist having as one set of lines of curvature general helices $(\rho / \tau=$ constant along each curve), but there are always limitations on the forms of $\rho$ and

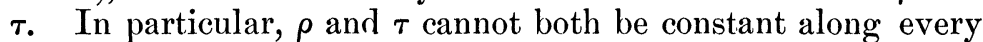
curve. The complete solution seems to be too wide for analytic discussion, but there are two particular cases for which definite results can be obtained. This note contains a discussion of these cases.

1) Assuming that $\rho$ and $\tau$ are constant along each curve of the set (regular helices), we obtain the result that: There are no surfaces with regular helices as one set of lines of curvature.

2) If $\rho / \tau$ is constant along each curve of one set of lines of curvature, and the other set consists of geodesics, we can obtain a complete solution; the equations of the resulting surfaces in parametric form are given at the end of this paper. The notation and equations used are those given in Darboux, Théorie des surfaces, volume 2 , but derivatives with respect to $u$ and $v$ are here denoted by suffixes 1 and 2 respectively.

\section{$\S 1$.}

Consider the case where the helices are all regular. The curves $v=$ constant are helices, and therefore $\rho$ and $\tau$ for these curves are functions of $v$ only, and we denote $\tau / \rho$ by $k$.

* For the suggestion of this subject I am indebted to Prof. A. R. Forsyth. $\dagger$ Messenger of Mathematics, vol. 38 (1908), pp. 33-44.

$\mp$ Note that $p_{1}$ is not $\partial p / \partial u$. To express derivatives of the rotations we use parentheses, e. $g \cdot(p)_{1}=\partial p / \partial u$. 
We have the following set of equations* :

$$
\begin{gathered}
p=0, \quad q_{1}=0 \\
\text { (1) } \frac{1}{\tau}=\frac{1}{A} \phi_{1}
\end{gathered}
$$

(2) $r=\frac{\sin \phi}{\rho} A, \quad\left(2^{\prime}\right) \quad r=-\frac{1}{C} A_{2}$,

(3) $q=-\frac{\cos \phi}{\rho} A, \quad$ (4) $p_{1}=\frac{1}{r}(q)_{2}$,

(5) $r_{1}=-\frac{1}{q}\left(p_{1}\right)_{1}, \quad\left(5^{\prime}\right) \quad r_{1}=\frac{1}{A} C_{1}$,

where $\phi$ is the angle between the osculating plane of the curve $v=$ constant and the normal plane to the surface. From (2), (3), (4), (5) we obtain

(7) $r_{1}=2 \frac{\phi_{12}}{k \phi_{1}} \sec \phi+\frac{k_{2}}{k^{2}} \operatorname{cosec}^{2} \phi \sec \phi-\frac{1}{k \phi_{1}}\left(\frac{\phi_{12} \operatorname{cosec} \phi}{\phi_{1}}\right)_{1}$

Also from (1), $\left(2^{\prime}\right),\left(5^{\prime}\right)$,

$$
r_{1}=\frac{1}{k}\left[\frac{\tau_{2}}{\tau} \cot \phi \operatorname{cosec} \phi-\frac{1}{\phi_{1}}\left(\frac{\phi_{12} \operatorname{cosec} \phi}{\phi_{1}}\right)_{1}\right] \text {. }
$$

From (7) and (8)

$$
\frac{\phi_{12}}{\phi_{1}}=\frac{1}{2}\left(\frac{\tau_{2}}{\tau} \cot ^{2} \phi-\frac{k_{2}}{k} \operatorname{cosec}^{2} \phi\right) .
$$

Differentiating with respect to $u$ and substituting in (8), we get

(9) $\quad r_{1}=\frac{3}{2} \cot \phi \operatorname{cosec} \phi\left[\operatorname{cosec}^{2} \phi\left(\frac{\tau_{2}}{\tau}-\frac{k_{2}}{k}\right)+\frac{\tau_{2}}{\tau}\right]$.

Combining (9) with (6) we obtain, after some reduction,

$$
\begin{aligned}
6 \operatorname{cosec}{ }^{4} \phi\left(\frac{\tau_{2}}{\tau}-\frac{k_{2}}{k}\right)- & \frac{3}{2} \operatorname{cosec}^{2} \phi\left(\frac{\tau_{2}}{\tau}-\frac{3 k_{2}}{k}\right) \\
+ & k^{2}\left[\operatorname{cosec}^{2} \phi\left(\frac{\tau_{2}}{\tau}-\frac{k_{2}}{k}\right)-\frac{\tau_{2}}{\tau}\right]=0 .
\end{aligned}
$$

* Darboux, 1. c., pages 383, 386. 
This equation determines $\phi$ as a function of $v$ only, but as the curves $v=$ constant are not plane curves, $\phi$ is a function of both $u$ and $v$, hence the equation must reduce to an identity. Equating to zero the coefficients of the various powers of $\operatorname{cosec} \phi$, we get

$$
\frac{\tau_{2}}{\tau}=0, \quad \frac{\tau_{2}}{\tau}-\frac{k_{2}}{k}=0,
$$

or $\rho=$ constant, $\tau=$ constant. Substituting in the preceding equations, we obtain finally $C=0$. Hence no surfaces exist of the kind we seek.

\section{$\S 2$}

Let us now assume that $\rho / \tau=\alpha$, a function of $v$ only, and that the curves $u=$ constant are geodesics. Having regard to the properties of geodesics, we may put $C=1$.

The equations referred to above now become

$$
\begin{array}{ll}
p=0, \quad q_{1}=0, \quad r_{1}=0, \\
\text { (i) } r=\frac{\sin \phi}{\rho} A, & \text { (i') } r=-A_{2}, \\
\text { (ii) } q=-\frac{\cos \phi}{\rho} A, & \text { (iii) }(r)_{2}+q p_{1}=0, \\
\text { (iv) } p_{1}=\frac{1}{r}(q)_{2}, & \text { (iv') } p_{1}=V . *
\end{array}
$$

There are two cases to be considered according as $V$ is zero or not. If $V$ is not zero, take a new variable $t$ so that $V d v=d t$. From (iii), (iv)

$$
\begin{aligned}
& \frac{\partial^{2} r}{\partial t^{2}}+r=0, \quad r^{2}+q^{2}=U^{\prime 2}, \\
& \therefore r=U^{\prime} \sin (t+U), \quad q=-U^{\prime} \cos (t+U) \text {. }
\end{aligned}
$$

From (i), (ii)

$$
t+U=\phi, \quad-A_{2}=U^{\prime} \sin (t+U) .
$$

We can absorb $U^{\prime}$ into $A$, which is equivalent to reducing $U^{\prime}$ to unity, and we obtain finally

\footnotetext{
* Capital letters $U, V$ denote functions of the variables $u$ alone, $v$ alone respectively.
} 


$$
A=\rho, \quad C=1, \quad \rho_{2}=-\sin (t+U), \quad U=\alpha u .
$$

The last result shows that $\alpha$, which is a function of $v$ only, must be an absolute constant; also $\rho$ must be of the form $a \cos \alpha u+b \sin \alpha u$, where $a$ and $b$ are functions of $v$ alone and

$$
a_{2}=-\sin t, \quad b_{2}=-\cos t .
$$

All the conditions are now satisfied, so that surfaces do exist having the required properties, and to obtain them we have the equations

$$
\begin{aligned}
x_{1}^{2}+y_{1}^{2}+z_{1}^{2} & =\rho^{2}, \quad x_{1} x_{2}+y_{1} y_{2}+z_{1} z_{2}=0, \quad x_{2}^{2}+y_{2}^{2}+z_{2}^{2}=1 . \\
D & =\rho^{2} \cos (t+k u), \quad D^{\prime}=0, \quad D^{\prime \prime}=\rho V^{*}
\end{aligned}
$$

These can be integrated and we obtain as coordinates of a point on a surface referred to a special set of axes

$$
\begin{gathered}
\left.x=\rho_{1} \frac{\sin \left(\sqrt{1+\alpha^{2}} \cdot u\right)}{\sqrt{1+\alpha^{2}}}-\rho \cos \sqrt{\left(1+\alpha^{2}\right.} \cdot u\right), \\
y=\rho_{1} \frac{\cos \left(\sqrt{1+\alpha^{2}} \cdot u\right)}{\sqrt{1+\alpha^{2}}}+\rho \sin \left(\sqrt{1+\alpha^{2}} \cdot u\right), \\
z=\frac{\rho_{1}}{\alpha_{\sqrt{1}} \cdot \alpha^{2}} .
\end{gathered}
$$

From a further consideration of the equation given on pages $383,386,1$. c., it appears that the curves $u=$ constant are plane curves, whose radii of curvature are given by

$$
1 / \rho=p_{1}=V .
$$

This shows that the set $u=$ constant consists of the different positions of an invariable curve in a plane which moves in a direction perpendicular to itself.

If $V=0$, we see at once that the surface is a developable whose coordinates may be written in the form

$$
x=a+l v, \quad y=b+m v, \quad z=c+n v,
$$

where $l, m, n, a, b, c$ are all functions of $u$ only, and in virtue

\footnotetext{
* Darboux, l. c., page 378.
} 
of the special properties of the curves $u=$ constant, $v=$ constant, satisfy the following relations :

$$
\begin{gathered}
l^{2}+m^{2}+n^{2}=1, \quad l_{1}^{2}+m_{1}^{2}+n_{1}^{2}=1, \\
\frac{a_{1}}{l_{1}}=\frac{b_{1}}{m_{1}}=\frac{c_{1}}{n_{1}}=\beta, \text { say. }
\end{gathered}
$$

Then we have $A=v+\beta$ and $d s^{2}=(v+\beta)^{2} d u^{2}+d v^{2}$. The equations to determine $p, q, r, p_{1}, q_{1}, r_{1}$, now become

$$
\begin{gathered}
p=0, \quad q_{1}=0, \quad r_{1}=0, \quad p_{1}=0, \\
\begin{array}{c}
\text { (1) } r=-1, \quad r=\frac{\sin \phi}{\rho}(v+\beta), \\
\text { (2) } q=U, \quad\left(2^{\prime}\right) \quad q=-\frac{\cos \phi^{\prime}}{\rho}(v+\beta), \\
\text { (3) } \tau \phi_{1}=v+\beta .
\end{array}
\end{gathered}
$$

Combining (1), $\left(1^{\prime}\right),(2),\left(2^{\prime}\right)$ we obtain

$$
\tan \phi=\frac{1}{U},
$$

which, upon differentiation with respect to $u$, and substitution from (3), leads to the following equation :

$$
\alpha=\frac{\rho}{\tau}=\frac{U_{1}}{\left(1+U^{2}\right)^{\frac{3}{2}}} .
$$

From this it is apparent that $\rho / \tau$, which is a function of $v$ only, is an absolute constant. By integration we obtain

$$
\frac{U}{\sqrt{1+U^{2}}}=\alpha u+\gamma \quad(\gamma=\text { constant }) .
$$

No generality is lost by making $\gamma=0$, and then

$$
U=\frac{\alpha u}{\sqrt{ } 1-\alpha^{2} u^{2}} .
$$

The equations to be integrated are 


$$
\begin{aligned}
& \sum l^{2}=1, \sum l_{1}^{2}=1, \quad \frac{l_{1}}{a_{1}}=\frac{m_{1}}{b_{1}}=\frac{n_{1}}{c_{1}}=\frac{1}{\beta}, \\
&\left|\begin{array}{lll}
l & m & n \\
l_{1} & m_{1} & n_{1} \\
l_{11} & m_{11} & n_{11}
\end{array}\right|=U
\end{aligned}
$$

and the surface is defined by the equations

$$
\begin{aligned}
& x=a+k u v, \\
& y=b+\frac{k u v}{U \sqrt{1-k^{2}}}(\cos \psi+k \sin \psi U), \\
& z=c+\frac{k u v}{U \sqrt{1-k^{2}}}(\sin \psi-k U \cos \psi),
\end{aligned}
$$

where $a, b, c$ are perfectly determinate functions of $u$, and

$$
k=\frac{\alpha}{\sqrt{1+\alpha^{2}}}, \quad \sin k \psi=\frac{k u}{\sqrt{1-k^{2}}} .
$$

Bryn Mawr College, Penna.,

December, 1908.

\title{
NOTE ON ENRIQUES'S REVIEW OF THE FOUNDATIONS OF GEOMETRY.
}

\author{
BY MR. A. R. SCHWEITZER.
}

(Read before the Chicago Section of the American Mathematical Society, A pril 18, 1908.)

1. As is well known, a continuous descriptive space may be extended to a projective one by the suitable definition of the projective points. For the definition of euclidean geometry on the basis of descriptive-projective geometry, we distinguish between the proper and improper points of the space and the assumption is made that all the improper points lie on the unique improper plane. This assumption may be provided for by means of a 